\section{Gategory}

Metal-Mediated

Synthesis

\section{Key words}

boron

cross-coupling

borazaronaphthalenes

G. A. MOLANDER,* J. AMANI, S. R. WISNIEWSKI (UNIVERSITY OF PENNSYLVANIA, PHILADELPHIA, USA)

Accessing 2-(Hetero)arylmethyl-, -allyl-, and -propargyl-2,1-borazaronaphthalenes: Palladium-Catalyzed CrossCouplings of 2-(Chloromethyl)-2,1-borazaronaphthalenes Org. Lett. 2014, 16, 6024-6027.

\title{
Accessing 2-(Hetero)arylmethyl-, -allyl-, and -propargyl-2,1-borazaronaphthalenes
}

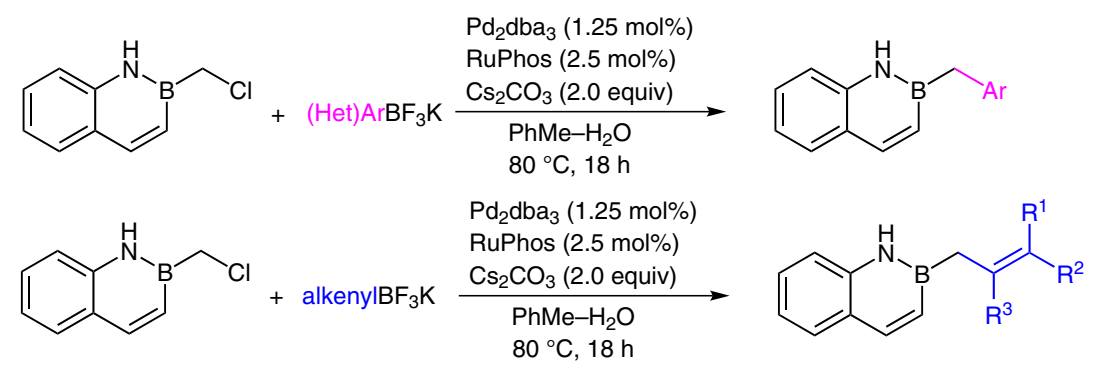

Selected examples:<smiles>C=Cc1cccc(CB2C=Cc3ccccc3N2)c1</smiles>

$80 \%$ yield<smiles>C/C=C/CB1C=Cc2ccccc2N1</smiles>

$90 \%$ yield<smiles>CC(=O)c1cccc(CB2C=Cc3ccccc3N2)c1</smiles>

$58 \%$ yield<smiles>Cc1ccc(CB2C=Cc3ccccc3N2)o1</smiles>

$67 \%$ yield<smiles>C1=Cc2ccccc2NB1Cc1ccsc1</smiles>

$70 \%$ yield<smiles>C=C(C)CB1C=Cc2ccccc2N1</smiles>

$80 \%$ yield<smiles>CCCCOC(=O)N1CC=C(CB2C=Cc3ccccc3N2)CC1</smiles>

$83 \%$ yield<smiles>C1=Cc2ccccc2NB1CC1=CCOCC1</smiles>

$79 \%$ yield
Significance: The authors expanded the electrophilic nature of 2-(chloromethyl)-2,1-borazaronaphthalene. In addition to substitution reactions, now also several metal-catalyzed reactions were performed. Potassium (hetero)aryl and alkenyl trifluoroborates as well as terminal alkynes were successfully used as nucleophiles.
Comment: Impressively, a wide variety of substituted azaborines were prepared starting from one common azaborinyl building block. This new methodology gives access to a whole library of pseudobenzylic-substituted azaborines. 